\title{
The impact of Gaia DR1 on asteroseismic inferences from Kepler
}

\author{
Travis Metcalfe ${ }^{1}$, Orlagh Creevey $^{2}$, and Jennifer van Saders ${ }^{3}$ \\ ${ }^{1}$ Space Science Institute, 4750 Walnut Street, Suite 205, Boulder CO 80301, USA \\ ${ }^{2}$ Université Côte d'Azur, Observatoire de la Côte d'Azur, CNRS, Laboratoire Lagrange, France \\ ${ }^{3}$ Carnegie Observatories, 813 Santa Barbara Street, Pasadena CA 91101, USA
}

\begin{abstract}
The Kepler mission has been fantastic for asteroseismology of solar-type stars, but the targets are typically quite distant. As a consequence, the reliability of asteroseismic modeling has been limited by the precision of additional constraints from highresolution spectroscopy and parallax measurements. A precise luminosity is particularly useful to minimize potential biases due to the intrinsic correlation between stellar mass and initial helium abundance. We have applied the latest version of the Asteroseismic Modeling Portal (AMP) to the complete Kepler data sets for 30 stars with known rotation rates and chromospheric activity levels. We compare the stellar properties derived with and without the measured parallaxes from the first data release of Gaia. We find that in most cases the masses and ages inferred from asteroseismology shift within their uncertainties. For a few targets that show larger shifts, the updated stellar properties only strengthen previous conclusions about anomalous rotation in middle-aged stars.
\end{abstract}

\section{Motivation}

The correlation between stellar mass and initial helium abundance is a long-standing problem in modeling solar-type stars. Increasing either the mass or the initial helium yields a model with a higher luminosity, so we can trade off one parameter for the other while still satisfying the observational constraints ([1]). Asteroseismic observations can reduce the severity of this problem by providing a strong constraint on the stellar radius, but the issue cannot be avoided entirely without a more direct constraint on the stellar luminosity. Without such a constraint, the correlation can lead to systematic biases in the stellar properties inferred from asteroseismology. In this paper, we examine the impact of including luminosity constraints derived from Gaia DR1 parallaxes ([2]) for a sample of $30 \mathrm{Kepler}$ targets with known rotation rates ([3]) and chromospheric activity levels ([4]). Our goal is to evaluate the possibility of systematic biases in the asteroseismic masses and ages for stars with anomalous rotation compared to empirical gyrochronology relations ([5]), with implications for a new theory of magnetic evolution beyond middle age ([6]).

Until recently, it was presumed that rotation and magnetism decay together throughout the lives of solar-type stars $([7,8])$. Although stars are formed with a range of initial rotation rates, the stellar winds entrained in their magnetic fields lead to angular momentum loss from magnetic braking. This forces convergence to a single rotation rate at a given mass after roughly 500 Myr. The Kepler mission allowed the measurement of rotation periods in old field stars whose masses and ages could be determined from asteroseismology. These new data revealed a population of field stars rotating 


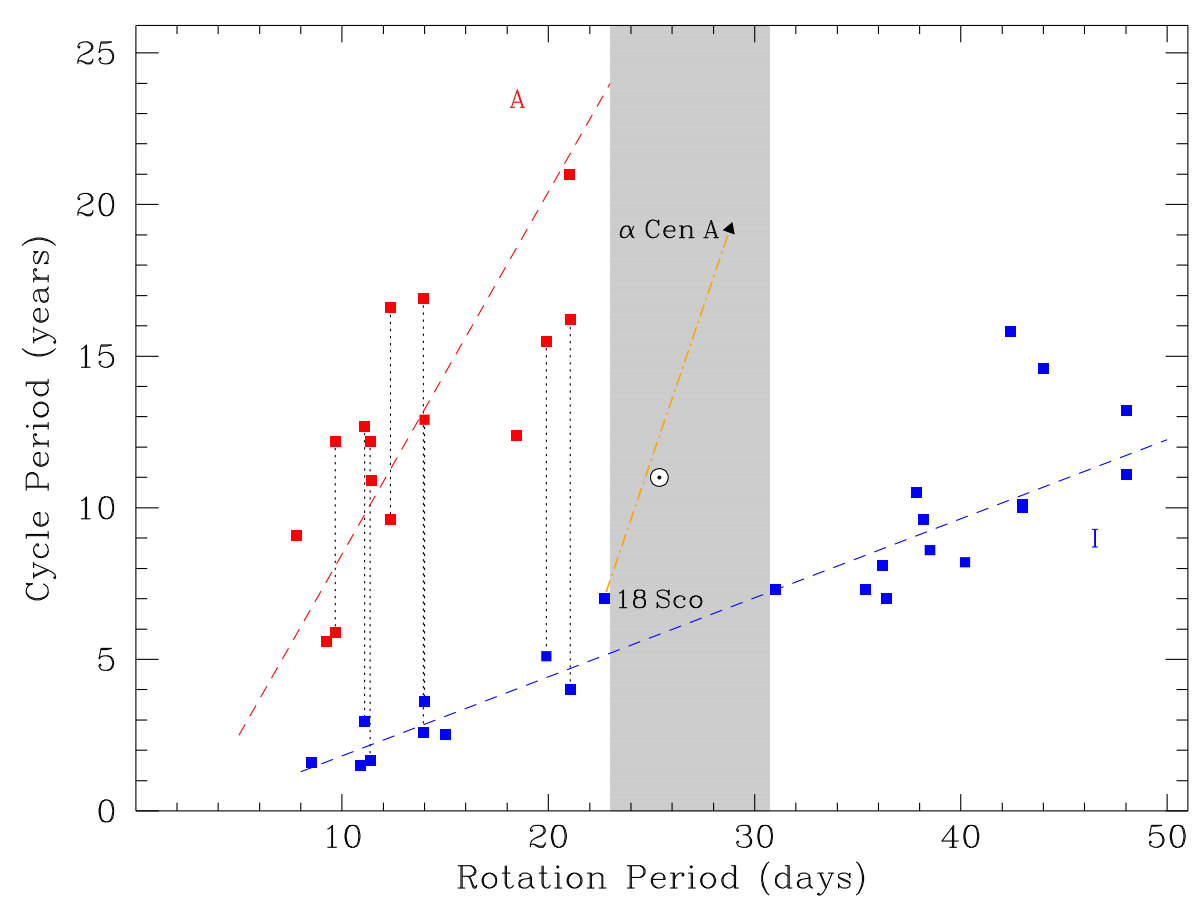

Figure 1. Updated version of a diagram published in [12] using data from [13], showing the active (A) and inactive (I) sequences. Multiple cycles observed in the same star are connected with vertical dotted lines. The shaded region indicates the rotation periods around the Sun $(\odot)$ where Mount Wilson stars do not show cycles.

more quickly than expected from gyrochronology ([9]), suggesting that magnetic braking may operate with a dramatically reduced efficiency beyond a critical Rossby number, Ro $\sim 2$ ([5]). A magnetic counterpart to this rotational transition was recently identified by [6]. They proposed that a change in the character of differential rotation is the underlying mechanism that ultimately disrupts the largescale organization of magnetic fields in solar-type stars. The process begins at Ro $\sim 1$, where many global convection simulations exhibit a transition from solar-like to anti-solar differential rotation ([10]). This change in the character of differential rotation triggers a phase of rapid magnetic evolution leading to a reduction in the efficiency of magnetic braking, probably due to a shift in magnetic topology ([11]).

The new picture of rotational and magnetic evolution provides a framework for understanding some observational features of stellar activity cycles that have until now been mysterious. An updated version of a diagram published in [12] is shown in Figure 1, using data from [13]. More recent data have been added from [14-20]. All of the slower rotators $\left(P_{\text {rot }}>30\right.$ days) are K-type stars, which is now understandable - magnetic braking ceases in more massive main-sequence stars before they reach these long rotation periods. The transition across Ro $\sim 2$ for G-type stars occurs at rotation periods comparable to the Sun ( $P_{\text {rot }} \sim 23-30$ days). If we consider the evolutionary sequence defined by $18 \mathrm{Sco}$, the Sun, and $\alpha$ Cen A, the data suggest that a normal cycle on the inactive sequence may grow longer across this transition (dot-dash line) before disappearing entirely. 


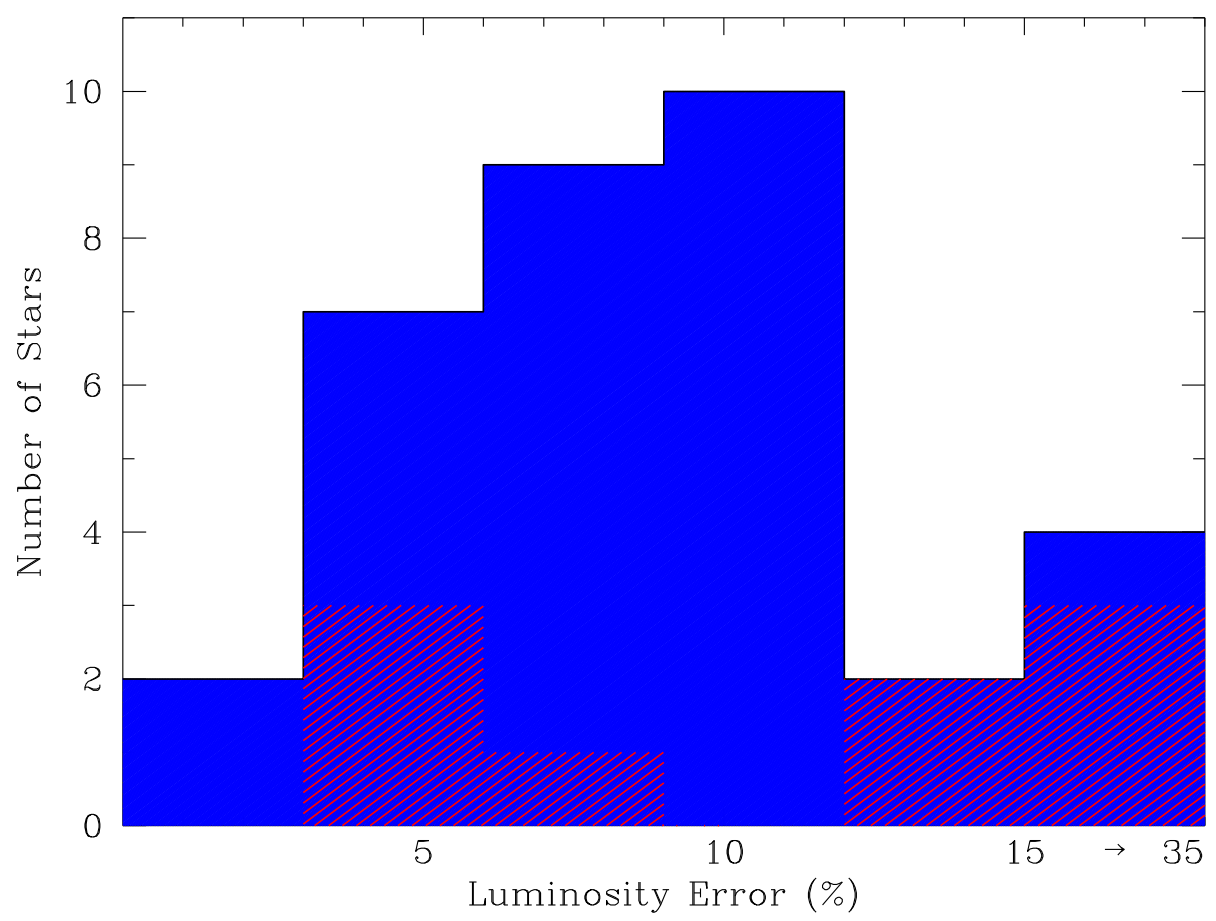

Figure 2. Histogram of the available luminosity constraints for our sample from the Tycho catalog (red) and from Gaia DR1 (blue). All of the stars in our sample now have luminosity constraints, and most have a precision better than $10 \%$. In late 2017, Gaia DR2 promises to improve the precision by nearly an order of magnitude.

\section{Asteroseismology with Kepler}

The Kepler space telescope yielded unprecedented data for the study of solar-like oscillations in other stars. Initial indications of anomalous rotation and magnetic activity in old field stars relied on asteroseismic properties from [21], which were based on an analysis of only 9 months of data ([22]). Kepler completed its primary mission in 2013, but the large samples of multi-year observations posed an enormous data analysis challenge that has only recently been surmounted ([23-25]). The longer data sets improved the signal-to-noise ratio $(\mathrm{S} / \mathrm{N})$ of the power spectrum for fainter stars with previously marginal detections, and yielded additional oscillation frequencies for the brighter targets. This expanded the asteroseismic sample of stars with known rotation rates ([3]) and chromospheric activity levels ([4]), from 21 targets ([26]) to more than 30.

We have used the latest version of the Asteroseismic Modeling Portal (AMP, [27]) to obtain reliable stellar properties from the complete Kepler data sets ([28]). For each star in the sample, we repeated the modeling with and without a luminosity constraint derived from Gaia DR1 parallaxes ([2]), allowing us to isolate the impact of this constraint on the inferred stellar properties. Parallaxes from the Tycho catalog were previously available for a few of the targets, but all of the stars in our sample now have luminosity constraints - and most have a precision better than $10 \%$ (see Fig. 2). 


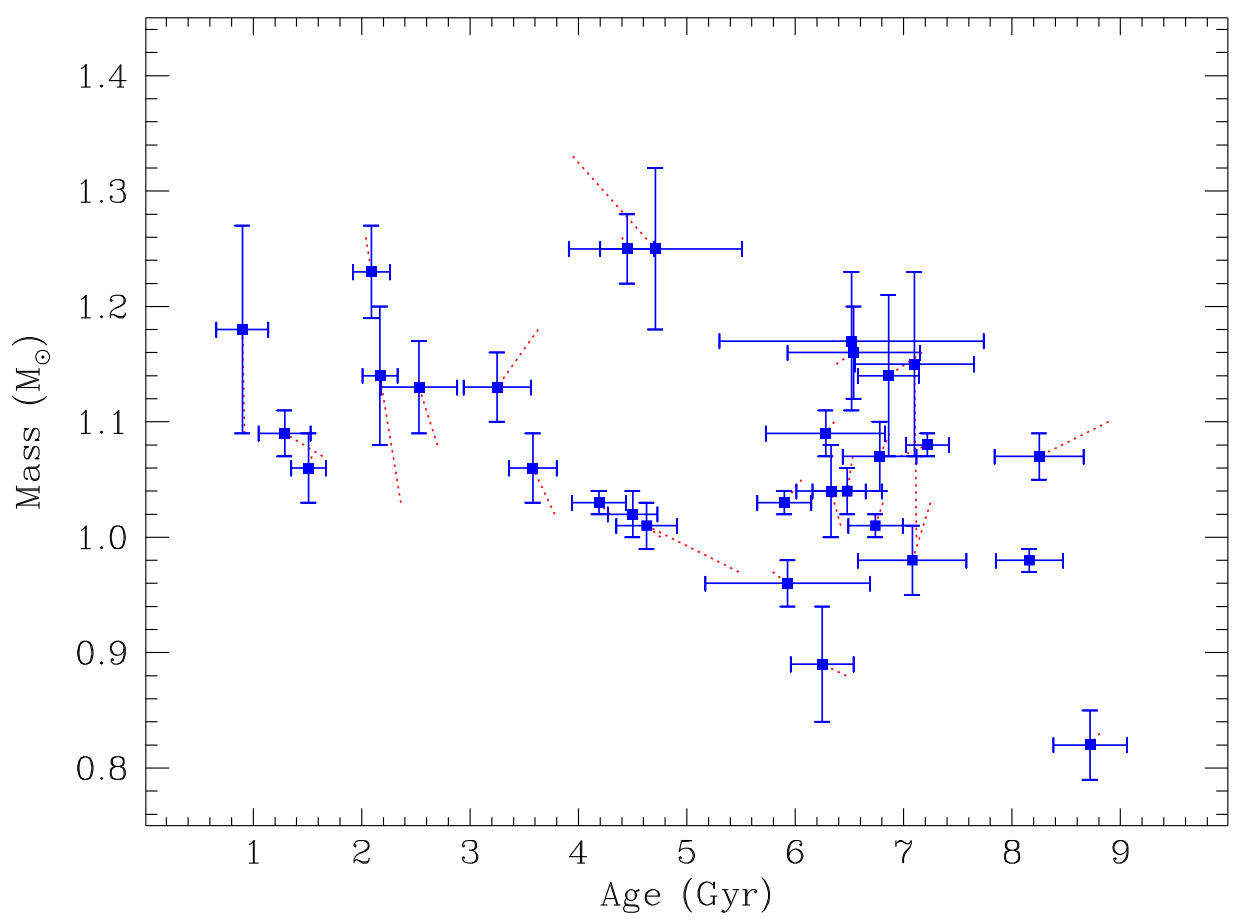

Figure 3. Stellar masses and ages inferred from asteroseismology before and after including luminosity constraints derived from Gaia DR1 parallaxes. Blue points with uncertainties show the values obtained when including a luminosity constraint, and dotted red lines connect to the values inferred without a luminosity constraint.

\section{Impact of Gaia parallaxes}

Claims of anomalous rotation in old field stars depend most directly on the inferred asteroseismic ages. The masses are also important because magnetic braking depends on the depth of the surface convection zone, and angular momentum evolution ultimately follows changes in the moment of inertia as a star expands into a subgiant. In Figure 3 we illustrate the impact of the available luminosity constraints on the inferred asteroseismic masses and ages. Most of the values shift within their respective uncertainties. For a few targets that show larger shifts, the updated stellar properties only strengthen previous conclusions about anomalous rotation in middle-aged stars.

In late 2017, Gaia DR2 promises to improve the parallax precision for all of our targets by nearly an order of magnitude. This will allow us to eliminate any remaining biases in the inferred stellar properties for our sample. It may also yield the asteroseismic composition with sufficient accuracy to distinguish between competing Galactic enrichment models.

Acknowledgments: This work was supported in part by NASA grants NNX15AF13G and NNX16AB97G, and by White Dwarf Research Corporation through the Non-profit Adopt a Star program. Computational time at the Texas Advanced Computing Center was provided through XSEDE allocation TG-AST090107. 


\section{References}

[1] Y. Lebreton, M.J. Goupil, A\&A, 569, A21 (2014)

[2] Gaia Collaboration, A.G.A. Brown, A. Vallenari, T. Prusti, J.H.J. de Bruijne, F. Mignard, R. Drimmel, C. Babusiaux, C.A.L. Bailer-Jones, U. Bastian et al., A\&A, 595, A2 (2016)

[3] R.A. García, T. Ceillier, D. Salabert, S. Mathur, J.L. van Saders, M. Pinsonneault, J. Ballot, P.G. Beck, S. Bloemen, T.L. Campante et al., A\&A, 572, A34 (2014)

[4] C. Karoff, T.S. Metcalfe, W.J. Chaplin, S. Frandsen, F. Grundahl, H. Kjeldsen, J. ChristensenDalsgaard, M.B. Nielsen, S. Frimann, A.O. Thygesen et al., MNRAS, 433, 3227 (2013)

[5] J.L. van Saders, T. Ceillier, T.S. Metcalfe, V. Silva Aguirre, M.H. Pinsonneault, R.A. García, S. Mathur, G.R. Davies, Nature, 529, 181 (2016)

[6] T.S. Metcalfe, R. Egeland, J. van Saders, ApJL, 826, L2 (2016)

[7] A. Skumanich, ApJ, 171, 565 (1972)

[8] S.A. Barnes, ApJ, 669, 1167 (2007)

[9] R. Angus, S. Aigrain, D. Foreman-Mackey, A. McQuillan, MNRAS, 450, 1787 (2015)

[10] T. Gastine, R.K. Yadav, J. Morin, A. Reiners, J. Wicht, MNRAS, 438, L76 (2014)

[11] V. Réville, A.S. Brun, S.P. Matt, A. Strugarek, R.F. Pinto, ApJ, 798, 116 (2015)

[12] E. Böhm-Vitense, ApJ, 657, 486 (2007)

[13] S.H. Saar, A. Brandenburg, ApJ, 524, 295 (1999)

[14] J.C. Hall, G.W. Henry, G.W. Lockwood, AJ, 133, 2206 (2007)

[15] L.E. DeWarf, K.M. Datin, E.F. Guinan, ApJ, 722, 343 (2010)

[16] T.S. Metcalfe, S. Basu, T.J. Henry, D.R. Soderblom, P.G. Judge, M. Knölker, S. Mathur, M. Rempel, ApJL, 723, L213 (2010)

[17] T.S. Metcalfe, A.P. Buccino, B.P. Brown, S. Mathur, D.R. Soderblom, T.J. Henry, P.J.D. Mauas, R. Petrucci, J.C. Hall, S. Basu, ApJL, 763, L26 (2013)

[18] T.R. Ayres, AJ, 147, 59 (2014)

[19] R. Egeland, T.S. Metcalfe, J.C. Hall, G.W. Henry, ApJ, 812, 12 (2015)

[20] D. Salabert, C. Régulo, R.A. García, P.G. Beck, J. Ballot, O.L. Creevey, F. Pérez Hernández, J.D. do Nascimento, Jr., E. Corsaro, R. Egeland et al., A\&A, 589, A118 (2016)

[21] T.S. Metcalfe, O.L. Creevey, G. Doğan, S. Mathur, H. Xu, T.R. Bedding, W.J. Chaplin, J. Christensen-Dalsgaard, C. Karoff, R. Trampedach et al., ApJS, 214, 27 (2014)

[22] T. Appourchaux, W.J. Chaplin, R.A. García, M. Gruberbauer, G.A. Verner, H.M. Antia, O. Benomar, T.L. Campante, G.R. Davies, S. Deheuvels et al., A\&A, 543, A54 (2012)

[23] G.R. Davies, W.J. Chaplin, W.M. Farr, R.A. García, M.N. Lund, S. Mathis, T.S. Metcalfe, T. Appourchaux, S. Basu, O. Benomar et al., MNRAS, 446, 2959 (2015)

[24] G.R. Davies, V. Silva Aguirre, T.R. Bedding, R. Handberg, M.N. Lund, W.J. Chaplin, D. Huber, T.R. White, O. Benomar, S. Hekker et al., MNRAS, 456, 2183 (2016)

[25] M.N. Lund, V. Silva Aguirre, G.R. Davies, W.J. Chaplin, J. Christensen-Dalsgaard, G. Houdek, T.R. White, T.R. Bedding, W.H. Ball, D. Huber et al., ApJ, 835, 172 (2017)

[26] T. Ceillier, J. van Saders, R.A. García, T.S. Metcalfe, O. Creevey, S. Mathis, S. Mathur, M.H. Pinsonneault, D. Salabert, J. Tayar, MNRAS, 456, 119 (2016)

[27] T.S. Metcalfe, O.L. Creevey, J. Christensen-Dalsgaard, ApJ, 699, 373 (2009)

[28] O.L. Creevey, T.S. Metcalfe, M. Schultheis, D. Salabert, M. Bazot, F. Thévenin, S. Mathur, H. Xu, R.A. García, A\&A, 601, A67 (2017), 1612.08990 